\title{
High Plastic Strain of Silica Microparticles under Electron Beam Irradiation
}

\author{
Douglas Stauffer, Sanjit Bhowmick, Ryan Major, Oden L. Warren and S. A. Syed Asif Hysitron Inc., \\ Minneapolis, Minnesota 55344, USA
}

The studies of irradiation damage in silica are of significant interest because of its application in nuclear reactors, nuclear waste containers, optical fibers, and semiconductor devices [1,2]. Although there are a number of publication showing the effect of electrons, ions, protons, alpha-particles irradiation on microstructural changes of silica, understanding deformation behavior under applied stress of irradiated sample is still lacking [2,3]. In this work, we investigate plastic flow and failure behavior of amorphous silica particles under compressive stress inside a scanning electron microscopy (SEM).

Silica microparticles (Cospheric LLC, Santa Barbara, CA) of diameter $1.050 \pm 0.030 \mu \mathrm{m}$ were mixed in ethanol, ultrasonicated for 10 minutes, and dispersed on silicon substrates. In situ quasistatic and dynamic compression experiments were conducted using a PI 85 SEM PicoIndenter (Hysitron, Inc., Minneapolis, MN) with $2.5 \mu \mathrm{m}$ flat punch diamond probe inside an SEM. The load-displacement data and the real-time video of deformation were synchronized and captured simultaneously, which aided the post-experimental analysis. Cyclic stress experiments were performed by applying a small oscillatory force $\left(P_{a}\right)$ at the peak force $\left(P_{\max }\right)$ and measuring the resulting phase, amplitude, and displacement. Single cycle quasistatic experiments were conducted at $P_{\max }=1 \mathrm{mN}$ and $4 \mathrm{mN}$ with a hold period of $190 \mathrm{~s}$ at peak load. Cyclic experiments were conducted at a frequencies (f) of 10 and 200 Hz with peak load $P_{\max }=1 \mathrm{mN}$ with oscillation load amplitude $P_{a}=0.1$.

The deformation behavior of the particles before and after the experiments with beam on and beam off conditions can be seen in figures a-f. A large variation in the total plastic strain and tendency to fracture has been observed which varies with peak loads and beam condition. Here, plastic strain has been calculated as the ratio $\delta / D$, where $D$ is the diameter of the particle and $\delta$ is the amount of compression along the indentation axis. In quasistatic experiments with a $190 \mathrm{~s}$ hold at $1 \mathrm{mN}$ peak load, a particle deformed plastically to $55.5 \%$ strain when the beam was kept on during the test (fig. a). However, when the beam was turned off (fig. b), a similar diameter particle showed negligible strain $(<0.05 \%)$. When the peak load is increased to $4 \mathrm{mN}$ peak load with the beam on, a plastic strain of $57.8 \%$ strain was found with a crack that appeared on the surface as marked in fig c. In beam off condition, a similar sphere deformed plastically to $37 \%$ strain, occurring in conjunction with a large fracture which created a wedge-shaped missing segment as observed in figure $d$. In all the fracture samples, the cracks tend to nucleate close to the initial contact zone and then propagate toward the periphery of the sphere leading to splitting of the particles. In summary, silica microparticles showed more plastic deformation with beam on condition compared to beam off condition. A similar deformation mechanism has been observed in cyclic loading with beam on and beam off conditions as shown in fig. e and f. At $P_{\max }=1$ $\mathrm{mN}$ with $P_{a}=0.1 \mathrm{mN}$, the sphere didn't show any measureable deformation with beam off condition whereas another sphere deformed plastically to $54.5 \%$ strain with beam on condition. The results in this study can be explained with the structural changes of the particles that has been reported in the literature $[1,4]$. It has been observed that electron beam with sufficient intensity can change the pore structure of amorphous silica where small pores shrink and larger pores expand [4]. The change in pore structure leads to softening of the particles which causes viscous fluid-type deformation. However, it should be emphasized that all the particles used in this study were exposed to electron beam before testing. So, it can be assumed that irradiation induced damage or defects in all the particles before loading were 
similar. This leads to a conclusion that the applied stress on the particles is playing a significant role in enhancing the structural changes and/or inducing more defects when electron beam is kept on. The deviation of the loading curves at a certain load in load-displacement plots in fig. $g$ and $h$ (marked by green arrows) also indicates that a minimum threshold stress is required to observe the stress-induced enhancement of plastic flow in this material. An important implication of this study is that electron irradiation under applied stress can induce significant instability and reduction in strength in silica resulting in lower lifetime in many devices where silica is an integral component.

\section{References:}

1. P. M. Ajayan and S. Iijima, Phil. Mag. Lett., 65 (1992), p. 43.

2. K. Zheng, Nature Communication, 1 (2010), p 1.

3. E. Snoeks, A. Polman and C. A. Volkert, APL, 65 (1994), p 112646.

4. A. J. Storm et al., J. of App. Phys., 98 (2005), p 014307.
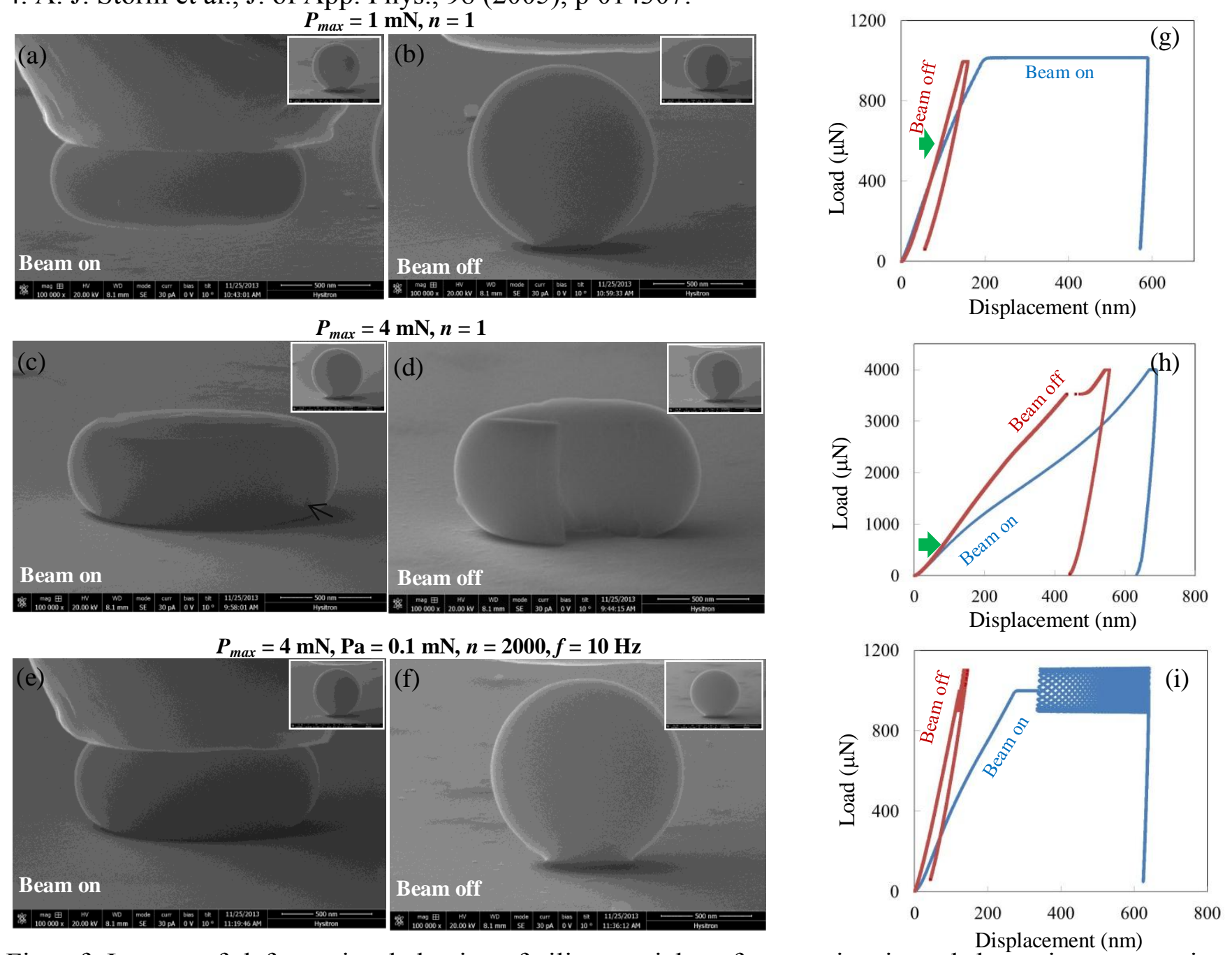

Fig a-f: Images of deformation behavior of silica particles after quasistatic and dynamic compression experiments with beam on and beam off conditions. $P_{\max }=$ peak load, $n=$ number of cycles, $P_{a}=$ load amplitude, $f=$ frequency. Fig g-h: Load-displacement plots at $P_{\max }=1$ and $4 \mathrm{mN}$ shows the effect of electron beam on plastic flow of the materials. Fig i: Load-displacement plots at $P_{\max }=1 \mathrm{mN}$ with $P_{a}=$ $0.1 \mathrm{mN}$ shows larger plastic strain under electron beam. 